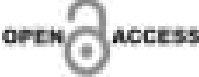

RESUMO DE ARTIGO

\title{
Artropatia por Deposição de Cristal Mimetizando Doença Inflamatória Sistêmica Febril em Paciente Idoso
}

\author{
Calcium Crystal-Associated Arthropathy Mimicking a Febrile \\ Systemic Inflammatory Disease in an Elderly Patient
}

\author{
Anna Paula Duque Sousa ${ }^{1}$, Carlos Moura ${ }^{1}$, \\ Priscila Ribeiro da Hora ${ }^{1}$, Mittermayer Santiago ${ }^{1}$ \\ ${ }^{1}$ Serviço de Reumatologia do Hospital Santa Izabel; Salvador, Bahia, Brasil
}

Correspondence addresses: Dr. Mittermayer Santiago mittermayer.santiago@ serdabahiacom.br

Received: July 30, 2019

Revised: August 17, 2019

Accepted: August 31, 2019

Published: September 27, 2019

Data Availability Statement: All relevant data are within the paper and its Supporting Information files.

Funding: This work was the result of authors' initiative. There was no support of research or publication funds.

Competing interests: The authors have declared that no competing interests exist.

Copyright

(C) 2019 by Santa Casa

de Misericórdia da Bahia.

All rights reserved.

ISSN: 2526-5563
A formação e deposição de cristais de cálcio nas articulações é uma causa importante e comum de artrite aguda. Raramente, a doença pode cursar com febre e sinais sistêmicos. Descrevemos o caso de um homem de 70 anos, que apresentou poliartrite súbita e incapacitante de grandes e pequenas articulações, febre, astenia e leucocitose. Após extensa investigação, a radiografia das articulações mostrou a presença de condrocalcinose. Poucos dias após o início do tratamento com colchicina, ele se tornou completamente assintomático, chamando a atenção da artropatia associada ao cristal de cálcio como causa de doença inflamatória sistêmica febril, particularmente na população idosa.

Palavras-chave: Cristais de Cálcio; Artrite Aguda; Doença Inflamatória Sistêmica Febril.

Crystal formation and deposition in the joints is an important and common cause of acute arthritis. The disease may present with fever and systemic signs. In this report, we describe the case of a 70-year-old man, who presented with a sudden and incapacitating polyarthritis of large and small joints, fever, asthenia and leukocytosis. After extensive investigation, radiography of the joints showed the presence of chondrocalcinosis. A few days after the beginning of the treatment with colchicine, he became completely asymptomatic, drawing one's attention of calcium crystal-associated arthropathy as a cause of febrile systemic inflammatory disease particularly in elderly population.

Keywords: Calcium Crystal; Acute Arthritis; Febrile Systemic Inflammatory Disease.

\section{Introdução}

A formação de cristais e a deposição de urato monossódico ou pirofosfato de cálcio nas articulações é uma causa importante e comum de artrite aguda. Em idosos, a doença de deposição de pirofosfato de cálcio di-hidratado (DPFC) é a principal causa de artrite aguda. Os grupos de maior risco para DPFC, além de idosos, são indivíduos com osteoartrite, história de trauma, distúrbios Resumo do Artigo: Anna Paula Duque Sousa, Carlos Moura, Priscila Ribeiro da Hora, and Mittermayer Santiago. Calcium crystal-associated arthropathy mimicking a febrile systemic inflammatory disease in an elderly patient. Oxford Medical Case Reports. Published online 2019 May 31. doi: 10.1093/omcr/omz030. 
genéticos e metabólicos, como hemocromatose, hiperparatireoidismo e hipomagnesemia. ${ }^{1}$ Quando a doença apresenta febre e sinais sistêmicos, pode levar a um diagnóstico incorreto e tratamento inadequado. Relatamos o caso de um paciente com poliartrite aguda de grandes e pequenas articulações associada à febre e sintomas infamatórios sistêmicos secundários a deposição de cristais de cálcio nas articulações.

\section{Relato do Caso}

Paciente do sexo masculino, 70 anos, internado no Hospital Santa Izabel, Salvador, Bahia, com febre e dor difusa, principalmente na região cervical e no ombro direito, iniciadas quatro dias antes da admissão. Logo após a internação, ele apresentou poliartrite de cotovelos, punhos, mãos, joelhos e tornozelos. Ao exame físico, o paciente estava acamado por causa da dor. Apresentava taquicardia, febre $\left(38^{\circ} \mathrm{C}\right)$ e poliartrite evidente no punho direito, joelhos, cotovelos, ombros, tornozelo direito e médio-pé direito. Demais exames físicos, normais. Exames laboratoriais revelaram hemoglobina de $14 \mathrm{mg} / \mathrm{dL}$, contagem total de leucócitos de 26.690 células $/ \mathrm{mm}^{3}$ sem desvio, velocidade de hemossedimentação de 120 $\mathrm{mm}$ e proteína C-reativa de $5,14 \mathrm{mg} / \mathrm{dL}$ (normal até $0,5 \mathrm{mg} / \mathrm{dL}$ ). Sorologias negativas para dengue, hepatite $\mathrm{B}$ e $\mathrm{C}$, vírus da imunodeficiência humana e sífilis. A cultura de urina foi negativa. O ácido úrico sérico estava dentro da faixa de normalidade e o paciente não tinha história de hiperuricemia. A função renal (cretinina $=0,7 \mathrm{mg} / \mathrm{dL}$; estimativa de clearance de creatinina por Cockcroft-Gault $=104,17 \mathrm{~mL} / \mathrm{min}$ ), eletrólitos (cálcio, fósforo e magnésio), bem como função tireoidiana e paratormônio normais. O ecocardiograma transesofágico não apresentou evidências de vegetação. Radiografias simples das articulações demonstraram condrocalcinose no menisco dos joelhos, fibrocartilagem dos punhos, articulações metacarpofalangeanas e cotovelos (Figura 1). O ombro direito apresentava estreitamento do espaço articular e irregularidade da superfície óssea glenoumeral, semelhante à observada na síndrome de Milwaukee (Figura 2). Infelizmente, não fomos capazes de realizar um estudo para definir o tipo de cristal presente em suas articulações porque a microscopia de luz polarizada para busca de cristal não estava disponível no setor. Um regime de $20 \mathrm{mg} /$ dia de prednisona foi iniciado para alívio sintomático da dor, com melhora apenas parcial. Assumindo um diagnóstico presuntivo de CCPD, colchicina $1 \mathrm{mg} /$ dia foi iniciado. $\mathrm{O}$ paciente apresentou melhora rápida da artrite e febre em 48 horas após a introdução do colchicina mesmo com a suspensão dos corticosteroides. Ele recebeu alta em uso de colchicina, andando normalmente sem febre e sem outras queixas.

\section{Discussão}

A DPFC é um diagnóstico diferencial importante de artrite aguda em idosos. Os locais mais comuns para a deposição de cristais são as fibrocartilagens e cartilagens hialinas (meniscos do joelho, cartilagem triangular do punho, articulação do quadril e glenoumeral). Quando os cristais são formados, eles podem interagir com a membrana das células plasmáticas, levando à liberação de IL-1 $\beta$ e esta citocina levará à ativação das células endoteliais, recrutamento de neutrófilos e liberação de outros mediadores inflamatórios, como o TNF- $\alpha$, o que pode contribuir para o desenvolvimento de artrite e manifestação sistêmica da DPFC. ${ }^{2}$

Os ataques agudos são autolimitados, semelhantes aos que ocorrem na gota aguda, razão pela qual o CPPD também é conhecido como pseudogota. Por outro lado, às vezes, a DPFC pode apresentar-se como poliartrite e pode estar associada a manifestações sistêmicas como febre, aumento dos níveis de reagentes de fase aguda e leucocitose, mimetizando doença infecciosa, como foi observado no presente caso. Além disso, alguns relatos de casos mostraram que a DPFC é uma causa de estado mental alterado em idosos, melhorando após o tratamento. ${ }^{3}$

Outra síndrome febril associada à deposição de cristais é chamada de síndrome do odontoide coroado, que é causada pela deposição de cristais 
ao redor do processo odontoide. Ela se manifesta como ataques recorrentes de febre, rigidez de nuca e é um importante diagnóstico diferencial para meningite, tumor espinhal ou espondilodiscite infecciosa. ${ }^{4}$

A síndrome de Milwaukee é um tipo artropatia destrutiva em ombros e que foi observada em nosso paciente. Esta tem sido classicamente relacionada à deposição de cristais básicos de fosfato de cálcio (BCP), mas também foi descrita em associação com a DPFC. Em nosso caso, a presença de condrocalcinose no menisco dos joelhos e na fibrocartilagem do punho corroboraria o diagnóstico de CPPD. No entanto, estudo realizado em cadáveres demonstrou que a condrocalcinose radiológica não é específica para a composição química do cristal, pois foi observada alta prevalência de cristais de PCB nas articulações com condrocalcinose. ${ }^{5}$ Assim, não podemos excluir a possibilidade da presença de dois tipos de cristais simultaneamente nas articulações de nosso paciente.

Em conclusão, o diagnóstico de poliartrite febril da DPFC em idosos baseia-se, em grande parte, na história clínica e exame musculoesquelético pelo médico assistente, além de aspiração articular e busca de cristais. Um estudo detalhado do líquido sinovial e do tipo de cristal neste cenário clínico é recomendado como parte da investigação da artrite inexplicada. Não ter realizado uma análise do líquido sinovial é uma limitação do nosso estudo. No entanto, a pesquisa de cristais através da microscopia de luz polarizada está disponível apenas em alguns poucos laboratórios especializados no Brasil. Por outro lado, do ponto de vista prático, a estratégia terapêutica é semelhante tanto para a deposição de cristais de CPPD como de BCP. De fato, a boa resposta à colchicina reforça o diagnóstico da doença para a deposição de cristais, mesmo não havendo discriminação do tipo de cristal.

\section{Referências}

1. Ea HK, Liote F. Diagnosis and clinical manifestations of calcium pyrophosphate and basic calcium phosphate crystal deposition diseases. Rheum Dis Clin North Am. 2014;40:207-229.
Figura 1. Radiografia simples mostrando condrocalcinose no menisco do joelho direito.

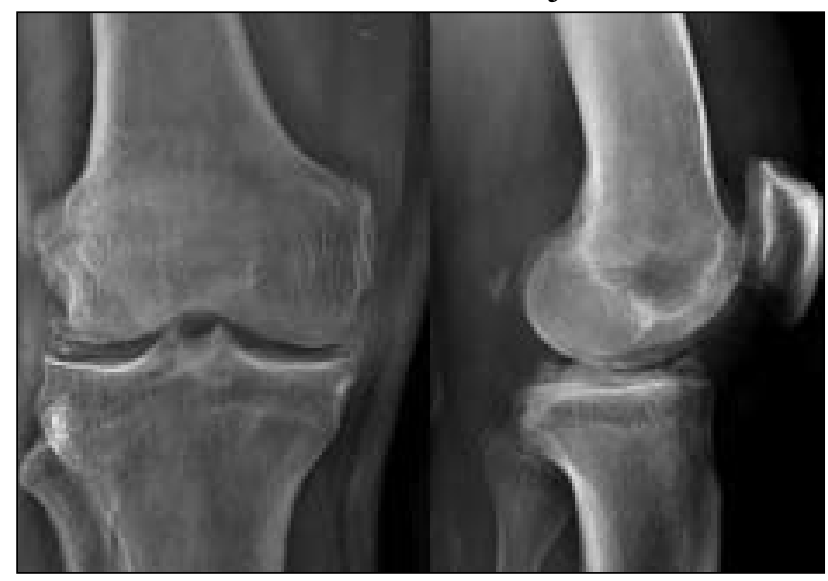

Figura 2. Radiografia simples do ombro direito revelando estreitamento do espaço articular e irregularidade da superfície óssea glenoumeral compatíveis com "ombro de Milwaukee".

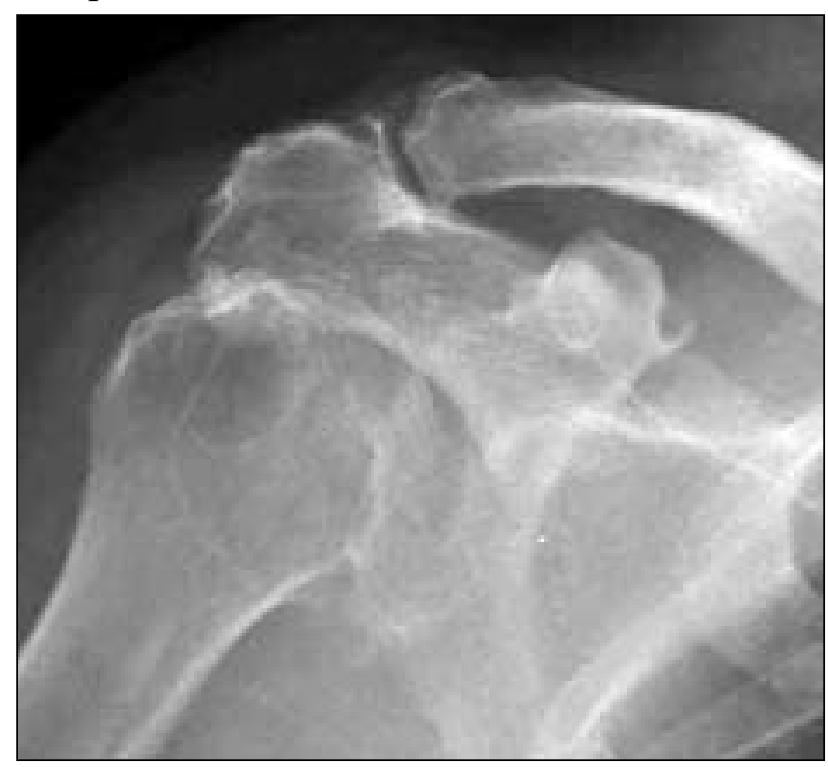

2. AbhishekA, Doherty M. Update on calcium pyrophosphate deposition. Clin Exp Rheumatol. 2016;34:32-38.

3. Kelley JT III, Agudelo CA, Sharma V, Holland NW. Fever with acute arthritis in calcium pyrophosphate dihydrate deposition disease: a missed explanation for altered mental status in elderly patients? J Clin Rheumatol. 2001;7: 322-325.

4. Godfrin-Valnet M, Godfrin G, Godard J, Prati C, Toussirot E, Michel $F$ et al. Eighteen cases of crowned dens syndrome: presentation and diagnosis. Neurochirurgie. 2013;59:115-120.

5. Liote F, Ea HK. Clinical implications of pathogenic calcium crystals. Curr Opin Rheumatol. 2014;26:192-196. 